\title{
On the Relation Between Black Hole Mass and Velocity Dispersion in Type 1 and Type 2 AGN
}

\author{
Kalliopi M. Dasyra ${ }^{1,2}$, Bradley M. Peterson ${ }^{3}$, Linda J. Tacconi ${ }^{4}$, \\ Hagai Netzer ${ }^{5}$, Luis C. Ho $^{6}$, George Helou ${ }^{1}$, Lee Armus ${ }^{1}$, \\ Dieter Lutz ${ }^{4}$, Richard Davies ${ }^{4}$, and Linda Watson ${ }^{3}$ \\ ${ }^{1}$ Spitzer Science Center, California Institute of Technology, USA \\ ${ }^{2}$ Service d' Astrophysique, Commissariat à l' Energie Atomique, France \\ ${ }^{3}$ Ohio State University, USA \\ ${ }^{4}$ Max Planck Institute for Extraterrestrial Physics, Germany \\ ${ }^{5}$ Wise Observatory, Israel \\ ${ }^{6}$ Carnegie Observatories, USA
}

\begin{abstract}
We present results from infrared spectroscopic projects that aim to test the relation between the mass of a black hole $M_{\mathrm{BH}}$ and the velocity dispersion of the stars in its host-galaxy bulge. We demonstrate that near-infrared, high-resolution spectroscopy assisted by adaptive optics is key in populating the high-luminosity end of the relation. We show that the velocity dispersions of mid-infrared, high-ionization lines originating from gas in the narrow-line region of the active galactic nucleus follow the same relation. This result provides a way of inferring $M_{\mathrm{BH}}$ estimates for the cosmologically significant population of obscured, type 2 AGN that can be applicable to data from spectrographs on next-generation infrared telescopes.
\end{abstract}

Keywords. galaxies: active, galaxies: nuclei, galaxies: kinematics and dynamics, (galaxies:) quasars: emission lines, infrared: galaxies, instrumentation: adaptive optics

\section{Introduction}

The comparison of the star-formation history of galaxies with the accretion rate of black holes (BHs) at different redshifts is of particular importance for our understanding of their evolution (e.g., Marconi et al. 2004; Netzer 2009). A challenge in this comparison is the determination of $\mathrm{BH}$ masses, $M_{\mathrm{BH}}$, in intermediate and high- $z$ galaxies, as well as in obscured AGN.

For nearby sources, $M_{\mathrm{BH}}$ can be inferred from stellar orbits (e.g., Kormendy et al. 1998; Genzel et al. 1998; Davies et al. 2006), gas or maser kinematics (e.g., Miyoshi et al. 1995), and reverberation experiments (Peterson et al. 2004). Most of these techniques often fail for sources at $z>0.5$ due to angular resolution and sensitivity limitations of the existing instrumentation. At such redshifts, $M_{\mathrm{BH}}$ is often estimated indirectly, using the relation between the mass of a $\mathrm{BH}$ and the velocity dispersion $\sigma$ of the stars in its galaxy bulge (Ferrarese \& Merritt 2000; Gebhardt et al. 2000; Gültekin et al. 2009). The stellar $\sigma$ value is typically substituted by the velocity dispersion of the narrow-line-region (NLR) gas clouds, which is measured from the [O III] $\lambda 5007$ line, assuming that the NLR kinematics are primarily determined by the gravitational potential of the bulge (Shields et al. 2003; Greene \& Ho 2005).

However, the obscuration of the optical [O III] line can be non negligible (Kauffmann et al. 2003). This can be a problem for NLR kinematic studies of obscured, type 2 sources, 
which constitute a cosmologically significant AGN population (e.g., Gilli et al. 2007; Lacy et al. 2007). We aimed to extend this technique in the MIR, where the NLR lines suffer little from obscuration. Moreover, the [Nev] and [O IV] lines have a higher ionization potential than [O III] and are therefore likely to originate from clouds that are closer to the $\mathrm{BH}$.

\section{The Existence of an $M_{\mathrm{BH}}-\sigma$ Relation for the NLR Gas Emitting in the MIR.}

We analyzed Spitzer IRS and ISO SWS archival data of local AGN with $M_{\mathrm{BH}}$ measurements from reverberation experiments (Peterson et al. 2004). We detected resolved $[\mathrm{NeV}] \lambda 14.32 \mu \mathrm{m}$ and [O IV] $\lambda 25.89 \mu \mathrm{m}$ lines in more than half of the sources in our sample (Dasyra et al. 2008). The resolution-corrected $\sigma$ measurements of both [Ne V] and [O IV] follow the $M_{\mathrm{BH}}-\sigma$ relation impressively well (Dasyra et al. 2008; see Figure 1 for $[\mathrm{NeV}]$ ) with a scatter that is comparable to that of the stellar velocity dispersions (Onken et al. 2004; Nelson et al. 2004), supporting previous findings that the NLR gas is often gravitationally bound to the bulge.

This result can have various applications for high-resolution MIR spectra from future IR observatories such as JWST and SPICA. Since the $M_{\mathrm{BH}}-\sigma$ relation holds for the NLR gas emitting in the MIR, it can be applied to distant and obscured sources with resolved MIR narrow lines to derive estimates of their BH masses. By comparing BH masses of type 1 and type 2 AGN at similar redshifts, it can also provide a testbed for the AGN unification model.

The flux of [Ne v] or [O IV] also correlates well with the optical $5100 \AA$ continuum flux (Schweitzer et al. 2006; Dasyra et al. 2008) for the observed AGN, indicating that it can be used as a proxy of the bolometric AGN luminosity, assuming standard bolometric conversion factors (Elvis et al. 1994; Marconi et al. 2004). Therefore, high-resolution MIR spectroscopy can also provide a means to derive the Eddington accretion rates for distant and obscured AGN.

\section{A Way to Improve the Calibration of the Local Stellar $M_{\mathrm{BH}^{-}} \sigma$ Relation}

In order to determine the accretion rate of BHs at intermediate and high- $z$ using gas kinematics and the $M_{\mathrm{BH}}-\sigma$ relation, it is necessary to test whether the relation remains identical with look-back time. Various studies in the literature suggest that an evolution of the relation could be possible (e.g., Salviander et al. 2007; Treu et al. 2007; Woo et al. 2008). However, determining or quantifying such an evolution remains highly uncertain. The limitations are primarily originating from the selection biases of distant sources, and from the lack of a robust calibration of the AGN $M_{\mathrm{BH}}-\sigma$ relation in high-luminosity AGN in the local Universe.

The sources that were initially used for the construction of the local AGN relation were primarily low-luminosity AGN. In QSOs, the absorption features that are traditionally used for the extraction of the stellar $\sigma$, such as the Ca II triplet at 8498, 8542, and $8662 \AA$ were undetected, heavily diluted by the bright AGN continuum. We demonstrated that in such cases the stellar $\sigma$ can be measured from the $H$-band CO bandheads (Dasyra et al. 2007). The advantage of using NIR instead of optical lines is that the AGN spectral energy distribution has a local minimum (Elvis et al. 1994) and the host galaxy has a local maximum close to $1.6 \mu \mathrm{m}$. The QSOs with $M_{\mathrm{BH}}$ and $\sigma$ measurements were located above the high-mass end of the relation (Dasyra et al. 2007; Watson et al. 2008; 




Figure 1. The relation between $M_{\mathrm{BH}}$ and the velocity dispersion of a NLR emission line in the MIR, [Nev], for reverberation-mapped AGN (Dasyra et al. 2008). The solid line corresponds to the best-fit solution to the $[\mathrm{NeV}]$ velocity dispersion values (excluding IC4329A) and the dashed line corresponds to the Tremaine et al. (2002) relation. The stellar velocity dispersion measurements of the same sources are plotted as stars (Onken et al. 2004; Nelson et al. 2004). Similar results are found for the [O IV] line width (Dasyra et al. 2008).

Figure 2). Differences in the kinematics of stellar populations alone could not account for the observed discrepancy.

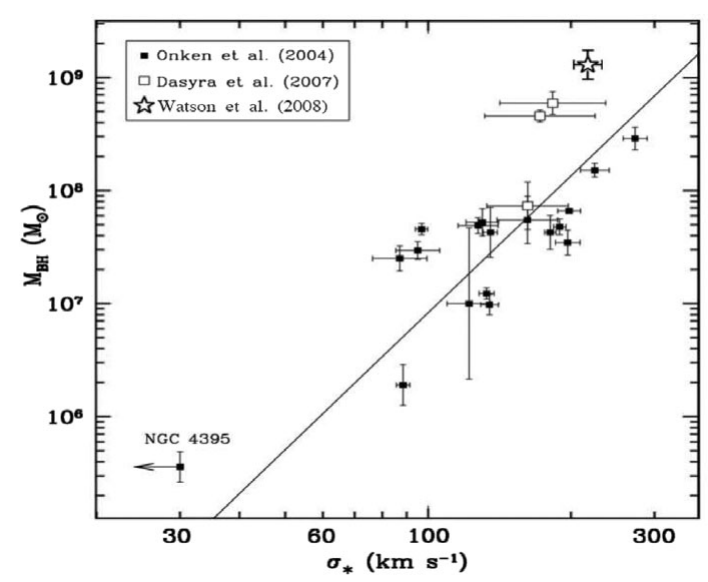

Figure 2. The AGN $M_{\mathrm{BH}}-\sigma$ relation, including the host galaxies of some reverberation-mapped QSOs. Adapted from Watson et al. (2008).

It is possible that the calibration of the BH masses in QSOs requires modifications. Specifically, the statistically determined value of the factor $f$ that converts the virial mass enclosed in the broad line region to the BH mass (Onken et al. 2004) could require a different prescription for AGN of high and low accretion rates (e.g., Collin et al. 2006). Alternatively, a steepening of the slope of the relation at the high luminosity or mass end is plausible, as it has been suggested based on [O III] kinematics (Gaskell 2009). Given the 
uncertainties in the slope of the local relation, its redshift evolution should be examined with caution.

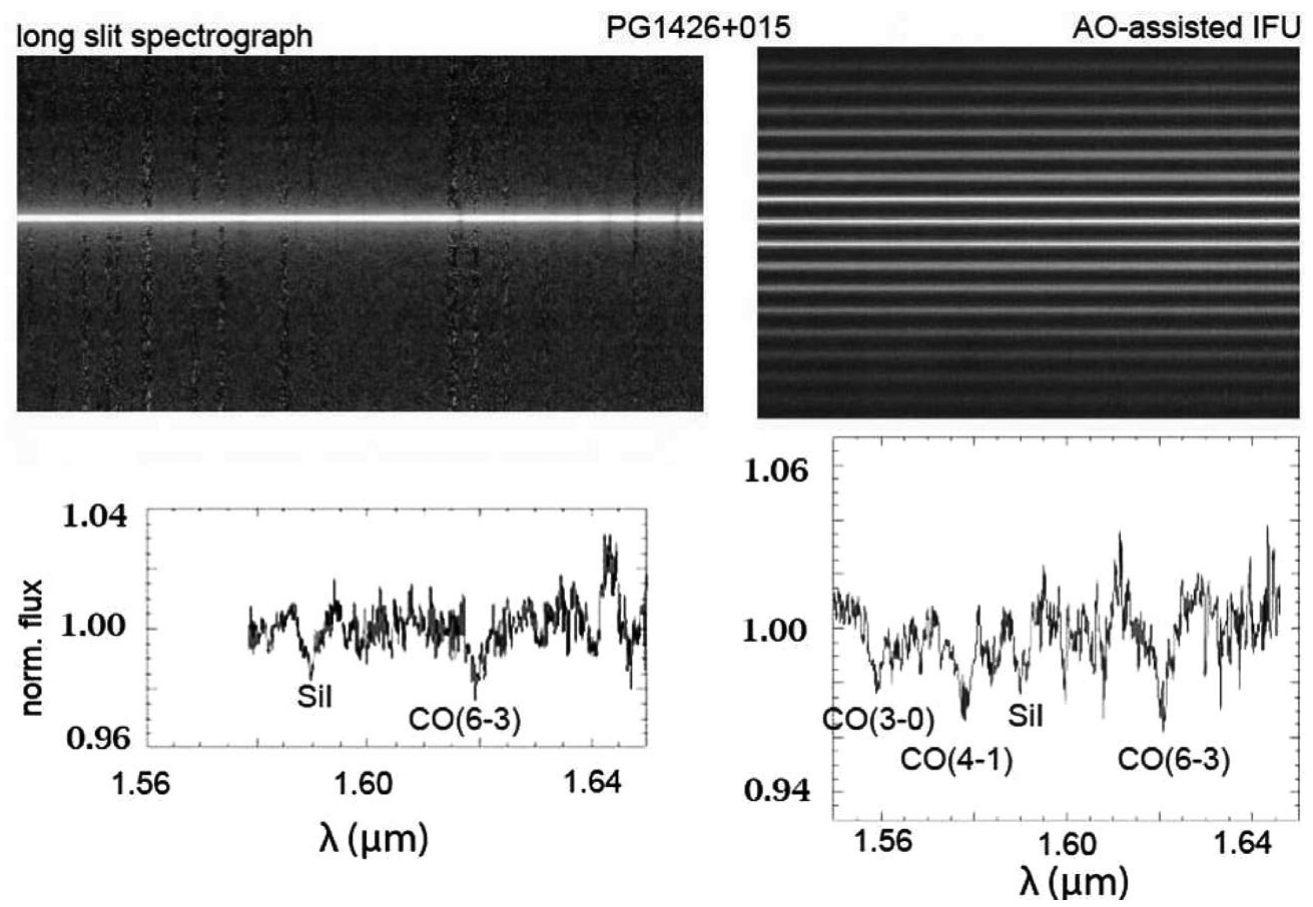

Figure 3. $H$-band spectra of PG $1426+015$, obtained with the long-slit spectrograph ISAAC at the VLT (left panels; data from Dasyra et al. 2007) and the IFU NIFS on GEMINI north (right panels; data used by Watson et al. 2008). The stellar CO absorption features are more prominent in the IFU data despite the $\sim 2$ times shorter on-source integration time.

To further study the behavior of the high-luminosity end of the relation, we have acquired NIR integral field unit (IFU) datasets with SINFONI and NIFS (Grier et al. these proceedings) that are assisted by adaptive optics. An example, PG 1426+015, was presented by Watson et al. (2008), which shows a significant improvement in $S / N$ ratio between the long-slit and the IFU spectra (Figure 3). The success of the IFU data relies in spatially disentangling the AGN from the host galaxy spectra (Figure 3 ).

\section{Conclusions}

The combination of Spitzer IRS and ISO SWS spectroscopy enabled the study of NLR gas kinematics in the MIR for reverberation-mapped AGN. The stellar $M_{\mathrm{BH}}-\sigma$ relation holds within the errors for NLR gas clouds that emit in the MIR as determined by the $[\mathrm{NeV}]$ and $[\mathrm{O} \mathrm{IV}]$ emission line widths. Therefore, resolved MIR narrow lines (in combination with the $M_{\mathrm{BH}}-\sigma$ relation) can be used to estimate the masses of BHs in obscured AGN. The measurement of stellar kinematics of QSO host galaxies can be achieved with NIR medium-to-high resolution long-slit and IFU spectroscopy. The hosts of local high-luminosity AGN have higher BH masses than those that the $M_{\mathrm{BH}}-\sigma$ relation would predict for their velocity dispersions, similar to what has been found for high- $z$ QSOs. This dictates the need for a better calibration of the relation prior to studying its possible evolution with $z$. 


\section{References}

Antonucci, R. R. J. \& Miller, J. S. 1985, ApJ, 297, 621

Collin, S., Kawaguchi, T., Peterson, B. M., \& Vestergaard, M. 2006 A\&A, 456, 75

Dasyra, K. M., et al. 2007, ApJ, 657, 102

Dasyra, K. M., et al. 2008, ApJ, 674, L9

Davies, R., et al. 2006, ApJ, 646, 754

Elvis, M., et al. 1994, ApJS, 95, 1

Ferrarese, L. \& Merritt, D. 2000, ApJ, 539, L9

Gaskell, C. M. 2009 [arXiv:0908.0328]

Gebhardt, K., et al. 2000, ApJ, 539, L13

Genzel, R., Pichon, C., Ekhart, A., Gerhard, O., \& Ott, T. 2000, MNRAS, 317, 348

Gilli, R., Comastri, A., \& Hasinger, G. 2007, A\&A, 463, 79

Greene, J. E. \& Ho, L. C. 2005, ApJ, 627, 721

Grupe, D. 2000, New Astron. Revs., 44, 455

Gültekin, K., et al. 2009, ApJ, 698, 198

Kauffmann, G., et al. 2003, MNRAS, 346, 1055

Kormendy, J., et al. 1998, AJ, 115, 1823

Lacy, M., et al. 2007, AJ, 133, 186

Marconi, A., Risaliti, G., Gilli, R., Hunt, L. K., Maiolino, R., \& Salvati, M. 2004, MNRAS, 351, 169

Miyoshi, M., Moran, J., Herrstein, J., Greenhill, L., Nakai, N., Diamond, P., \& Inoue, P. 1995, Nature, 373, 127

Nelson, C., Green, R. F., Bower, G., Gebhardt, K., \& Weistrop, D. 2004, ApJ, 615, 652

Netzer, H. 2009, MNRAS, 399, 1907

Onken, C. A., Ferrarese, L., Merritt, D., Peterson, B. M., Pogge, R. W., Vestergaard, M., \& Wandel, A. 2004, ApJ, 615, 645

Peterson, B. M., et al. 2004, ApJ, 613, 682

Salviander, S., Shields, G., Gebhardt, K., \& Bonning, E. 2007, ApJ, 662, 131

Shields, G. A., Gebhardt, K., Salviander, S., Wills, B. J., Xie, B., Brotherton, M. S., Yuan, J., \& Dietrich, M. 2003, ApJ, 583, 124

Schweitzer, M., et al. 2006, ApJ, 649, 79

Tremaine, S., et al. 2002, ApJ, 574, 740

Treu, T., Woo, J.-H., Malkan, M., \& Blandford, R. 2007, ApJ, 667, 117

Watson, L., et al. 2008, ApJ, 682, L21

Woo, J.-H., Treu, T., Malkan, M., \& Blandford, R. 2008, ApJ, 681, 925 